\title{
Side Chain Motion of Poly(L-glutamate)s Having Long Alkyl Side Chains as Studied by Spin-Probe Technique
}

\author{
Masashi Yamaguchi, Masao TezuKa, ${ }^{*}$ and Akihiro Tsutsumi \\ Department of Applied Physics, Faculty of Engineering, \\ Hokkaido University, Sapporo 060, Japan
}

(Received June 25, 1990)

\begin{abstract}
The side chain motion of $\operatorname{copoly}(\gamma$-methyl L-glutamate-co- $\gamma$ - $n$-alkyl L-glutamate) was studied by a spin-probe technique to examine its dependence on the copolymer composition and alkyl side chain length. In a copolymer series with alkyl side chain length $n=12$ and 15 (where $n$ is number of alkyl carbon), there is a tendency that side chain motion becomes rapid as the degree of substitution of long alkyl side chain (D.S.) increases. At the highest D.S., a copolymer with $n=15$ forms crystallites composed of long alkyl chains and showed a sudden decrease in the rotational correlation time of spin-probes around the crystalline melt, whereas a copolymer with $n=12$ did not show such a decrease owing to the smaller side chain crystallite. Correlation time-temperature profile for copoly $(\gamma$-benzyl L-glutamate-co- $\gamma$-stearyl L-glutamate) $(n=18)$ and that previously reported for copoly $(\gamma$-methyl L-glutamate-co- $\gamma$-stearyl L-glutamate) $(n=18)$ were quite similar at comparable D.S. except for a small D.S. region. This means that motion of long alkyl side chain is not affected by short side chains.

KEY WORDS Poly(glutamate)s / Copolymer / Long Alkyl Side Chain /

Spin-Probe Technique / Molecular Motion /
\end{abstract}

It has been reported that polypeptides with long alkyl side chains $(n \geqq 10: n$ is number of alkyl carbon atoms) have characteristic features not observed in poly(glutamate)s with short side chains ${ }^{1-3}$; that is, the alkyl side chains form paraffin-like crystallites and at a certain temperature above the crystalline melt, thermotropic liquid crystalline nature appears. It has been suggested ${ }^{1,4,5}$ that the formation of such a liquid crystal is closely related to considerably high mobility of the long alkyl side chains. It seems therefore important to reveal the dependence of side chain structure and molecular motion of poly(glutamate)s on alkyl side chain length in more detail. ${ }^{1,4}$

In the previous paper, ${ }^{5}$ we investigate the structures and dynamical properties of $\operatorname{poly}(\gamma-$ methyl L-glutamate-co- $\gamma$-stearyl L-glutamate) (St copolymer, $n=18$ ) with various degrees of substitution (D.S.) by means of electron spin resonance (ESR) using a spin-probe technique and ${ }^{13} \mathrm{C}$ cross polarization/magic angle spinning nuclear magnetic resonance (CP/MAS NMR). It was found that the side chain region surrounding the $\alpha$-helical main chain is classified into three parts in terms of mobility; 1) inner part composed of methyl glutamate (MLG) side chain and inner portion of stearyl glutamate (STLG) side chain, 2) amorphous part of stearyl group, and 3) crystalline part of stearyl group. The motion of inner part is constrained and relatively slow, whereas the amorphous part of stearyl group undergoes very rapid motion.

It has been well known that the spin-probe technique is useful to investigate the molecular motion of the host polymers where small amounts of probes such as nitroxide radicals

* Present address: TDK Co. Ltd., Ichikawa, Japan 
are dispersed. ${ }^{6,7}$ Especially, for $\alpha$-helical poly(glutamate)s, this technique gives motional information in the side chain region selectively where nitroxide radicals are embedded. ${ }^{5}$

In this study, we employed this technique to obtain further information on side chain motion in two series of copolymers with different alkyl side chain length; poly $(\gamma$-methyl L-glutamate-co- $\gamma$-pentadecyl L-glutamate) ( $n=$ 15, PD copolymer) and poly $(\gamma$-methyl Lglutamate-co- $\gamma$-lauryl L-glutamate) $(n=12$, LA copolymer). We also investigated a series of poly $(\gamma$-benzyl L-glutamate- $c o$ - $\gamma$-stearyl L-glutamate) ( $n=18$, STB copolymer) to see the effects of different short side chain units on the mobility of long alkyl groups by comparing the previous results for ST copolymers with $n=18$ containing MLG as a short side chain unit.

\section{EXPERIMENTAL}

\section{Materials}

Poly( $\gamma$-methyl L-glutamate) (PMLG) was kindly supplied by Ajinomoto Co., Ltd. PD and LA copolymers were synthesized by ester exchange reaction of PMLG with respective alcohol in 1,2-dichloroethane at $60^{\circ} \mathrm{C}$ using $p$-toluenesulfonic acid as a catalyst. The degree of substitution of $n$-alkyl group (mol\%), D.S., in copolymers was adjusted by changing the alcohol concentration and confirmed by ${ }^{1} \mathrm{H}$ NMR in a deuterated chloroform-trifluoroacetic acid $(80: 20 \%)$ solution. STB copolymer was synthesized by the ester exchange of poly $(\gamma$-benzyl L-glutamate) (PBLG) and stearyl alcohol with the similar conditions as described above. PBLG was obtained by the ester exchange reaction of PMLG with benzyl alcohol. All samples examined were films prepared by casting the chloroform solution at room temperature. Abbreviations for samples are listed in Table I.

For ESR measurement, 2,2,6,6-tetramethyl4-hydroxypiperidine-1-oxyl (TEMPOL) was used as a spin-probe (Figure 1). The film was immersed in a $10^{-3} \mathrm{~mol}^{-1}$ ethanol solution

Table I. Abbreviations for samples

\begin{tabular}{lcccc}
\hline Abbr. & Short side chain unit $^{\mathbf{a}}$ & Long side chain unit $^{\mathbf{b}}$ & $(n)^{\mathbf{c}}$ & D.S. $^{\mathbf{d}}$ \\
\hline PD92 & MLG & PDLG & $(15)$ & 92 \\
PD69 & MLG & PDLG & $(15)$ & 69 \\
PD64 & MLG & PDLG & $(15)$ & 64 \\
PD54 & MLG & PDLG & $(15)$ & 54 \\
PD31 & MLG & PDLG & $(15)$ & 31 \\
LA98 & MLG & LALG & $(12)$ & 98 \\
LA56 & MLG & LALG & $(12)$ & 56 \\
LA51 & MLG & LALG & $(12)$ & 51 \\
LA15 & MLG & LALG & $(12)$ & 15 \\
STB85 & BLG & STLG & $(18)$ & 85 \\
STB58 & BLG & STLG & $(18)$ & 58 \\
STB20 & SLG & STLG & $(-)$ & 20 \\
PBLG & BLG & STLG & $(18)$ & 0 \\
ST83* & MLG & STLG & $(18)$ & 83 \\
ST62* & MLG & STLG & $(18)$ & 62 \\
ST38* & MLG & STLG & $(18)$ & 38 \\
ST25* & MLG & - & $(-)$ & 25 \\
PMLG* & MLG & & & 0 \\
\hline
\end{tabular}

a MLG, $\gamma$-methyl L-glutamate; BLG, $\gamma$-benzyl L-glutamate.

b PDLG, $\gamma$-pentadecyl L-glutamate; LALG, $\gamma$-lauryl L-glutamate; STLG, $\gamma$-stearyl L-glutamate.

c Number of carbons in long alkyl chain.

d Degree of substitution.

* Previous work. ${ }^{5}$ 


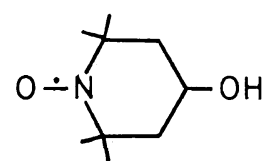

Figure 1. Chemical structure of TEMPOL.

of the probe for $12 \mathrm{~h}$ and dried in vacuo.

\section{Measurements}

ESR spectra were taken with a JEOL X-band $(9.2 \mathrm{GHz})$ spectrometer (JES-FE1X) at a microwave power of $1 \mathrm{~mW}$. The amplitude of $100 \mathrm{kHz}$ field modulation was $0.3-0.63$ gauss. These are the conditions for no saturation and modulation broadning. The temperature was varied from -130 to $150^{\circ} \mathrm{C}$.

Differential scanning calorimetric (DSC) measurements were performed at a scanning rate of $5 \% \mathrm{~min}$ in heating process using a Rigaku-Denki DSC S-type calorimeter.

\section{RESULTS AND DISCUSSION}

\section{D.S. Dependence of Spin-Probe Motion}

D.S. Dependence of ESR Line Shape. Typical examples of ESR spectra for TEMPOL in PD64 and PMLG at various temperatures are shown in Figure 2. Spectral line shapes for both samples change from a broad and asymmetric pattern to a sharp triplet pattern with increasing temperature. Such changes are attributed to changes in the motional state of TEMPOL, which is governed by the motional state of the environment, ${ }^{6}$ the side chain region surrounding the rigid $\alpha$-helical main chains. ${ }^{8}$ PD64 has sharper spectra than PMLG at higher temperatures, indicating that the mobility in the side chain region for the former is higher than that for the latter. Other PD copolymers showed spectra similar to those of PD64. This means that the introduction of PD group raises the mobility of the side chain region. For LA and STB copolymer series, similar results were obtained.

The spectral line narrowing temperature is defined as $T_{50 \mathrm{G}}$ where the extrema separation

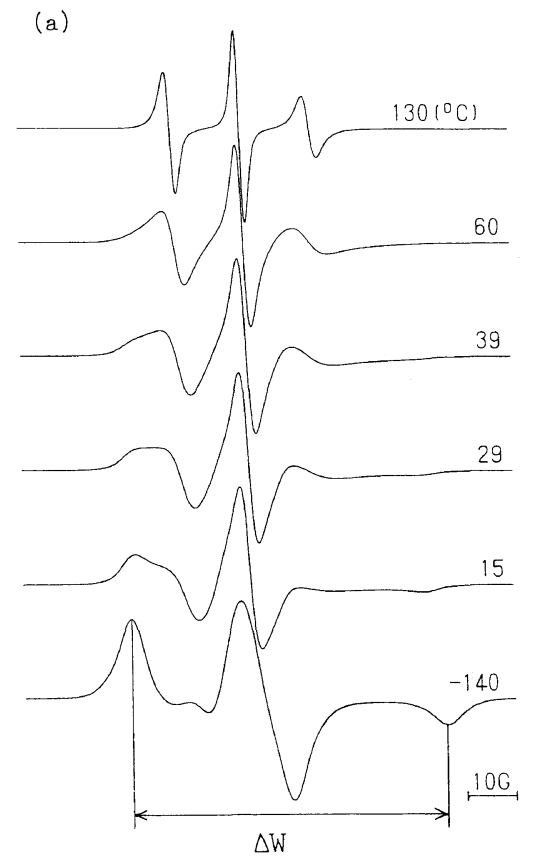

(1)

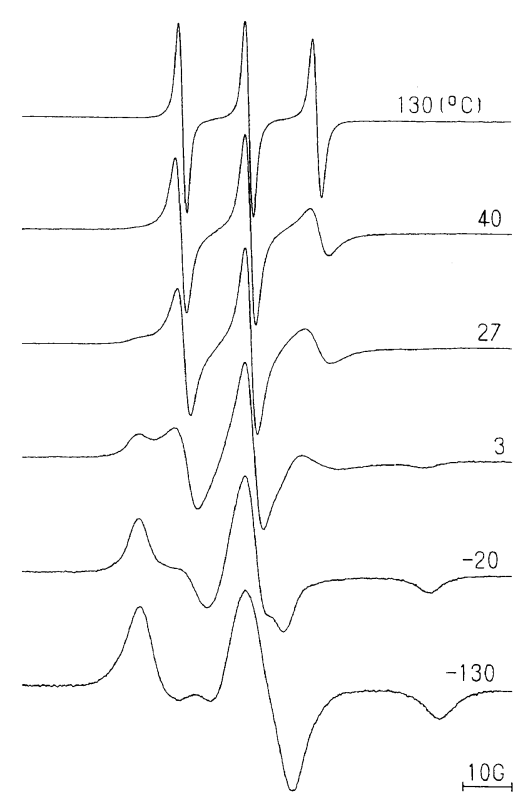

Figure 2. ESR spectra of TEMPOL in PMLG (a) and PD64 (b).

$\Delta W$ crosses 50 Gauss and rotational correlation time of TEMPOL becomes $c a .10^{-8} \mathrm{~s} .{ }^{9}$ In Figure 3, $T_{50 \mathrm{G}}$ is plotted as a function of D.S. 

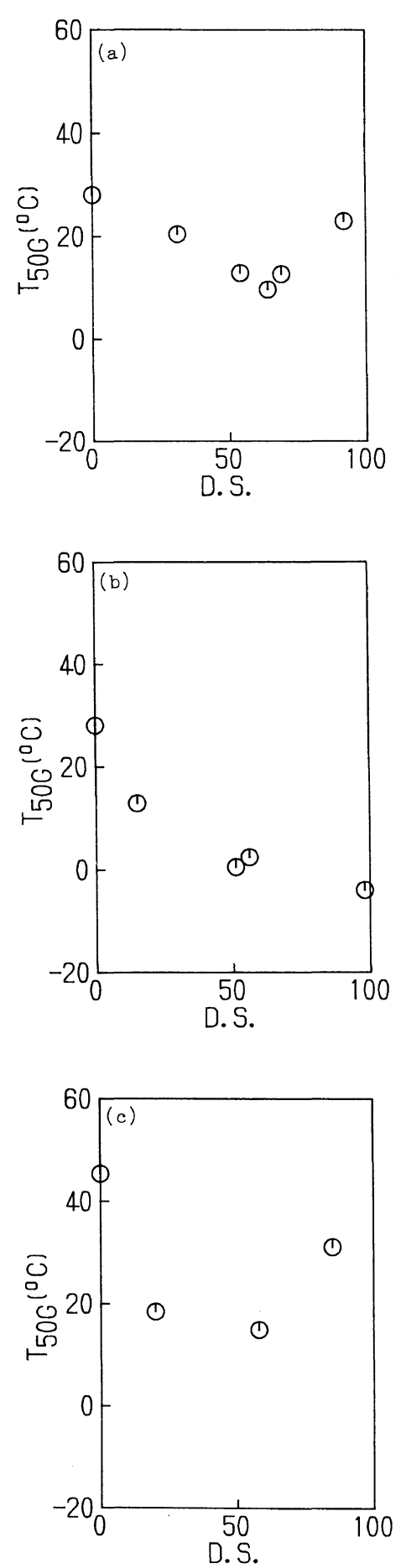

Figure 3. $T_{50 \mathrm{G}} v s$. D.S. for PD (a), LA (b), and STB (c) copolymers.
In all series of copolymers, an introduction of long alkyl group lowers the $T_{50 \mathrm{G}}$ compared to that of PMLG (D.S. $=0$ ). In the intermediate region of D.S., $T_{50 \mathrm{G}}$ shows a tendency to keep the lowest value. However, $T_{50 \mathrm{G}}$ rises more than $10^{\circ} \mathrm{C}$ in the copolymer with highest D.S. except for LA copolymers having the shortest alkyl chain among the copolymers examined in this work. Detailed discussion about this point is given below.

D.S. Dependence of Correlation Times for Spin Probe Motion. To obtain further detailed information on the motional state of the spin-probe, the correlation time of the rotational diffusion, $\tau_{c}$, was estimated from the ESR spectra. For the fast tumbling region $\left(10^{-9}<\tau_{\mathrm{c}}<10^{-11} \mathrm{~s}\right)$, the procedures by Freed et al., ${ }^{6,10}$ which assumes axially symmetric ellipsoidal motion of spin-probe, was employed. The average correlation time, $\tau_{\mathbf{c}}=$ $\sqrt{\tau_{\|} \tau_{\perp}}, 11$ was defined as that for isotropic motion, where $\tau_{\|}$and $\tau_{\perp}$ are the correlation times for the rotational diffusion about major and minor axes of ellipsoid, respectively. For the slow tumbling region $\left(10^{-7}<\tau_{\mathrm{c}}<10^{-9} \mathrm{~s}\right)$, the relation derived from the simulated spectra by Freed et al. ${ }^{12}$ was used, assuming a Brownian motion model with a intrinsic line width of 3 gauss.

$$
\tau_{\mathrm{c}}=a(1-S)^{b}, \quad \text { where } \quad S=A_{\mathrm{Z}} / A_{\mathrm{zrigid}}
$$

$A_{\mathrm{z}}$ is the half width of extrema separation and $A_{\text {zrigid }}$ is its rigid limit value. $a$ and $b$ are constants and have values of $5.4 \times 10^{-10}$ and -1.36 , respectively, for the conditions of this work. More detailed descriptions appear in our previous paper. $^{5}$

The temperature dependence of $\tau_{\mathrm{c}}$ for PD, LA, and STB copolymers is shown in Figure 4. Each sample does not show a simple Arrhenius type linear $\log \tau_{\mathrm{c}}-1000 / T(\mathrm{~K})$ relationship over the whole temperature range, indicating that motional process is not single. Quite similar results were obtained for ST copolymers. ${ }^{5}$ The arrows in the figures indicate the region where the sudden decrease of 

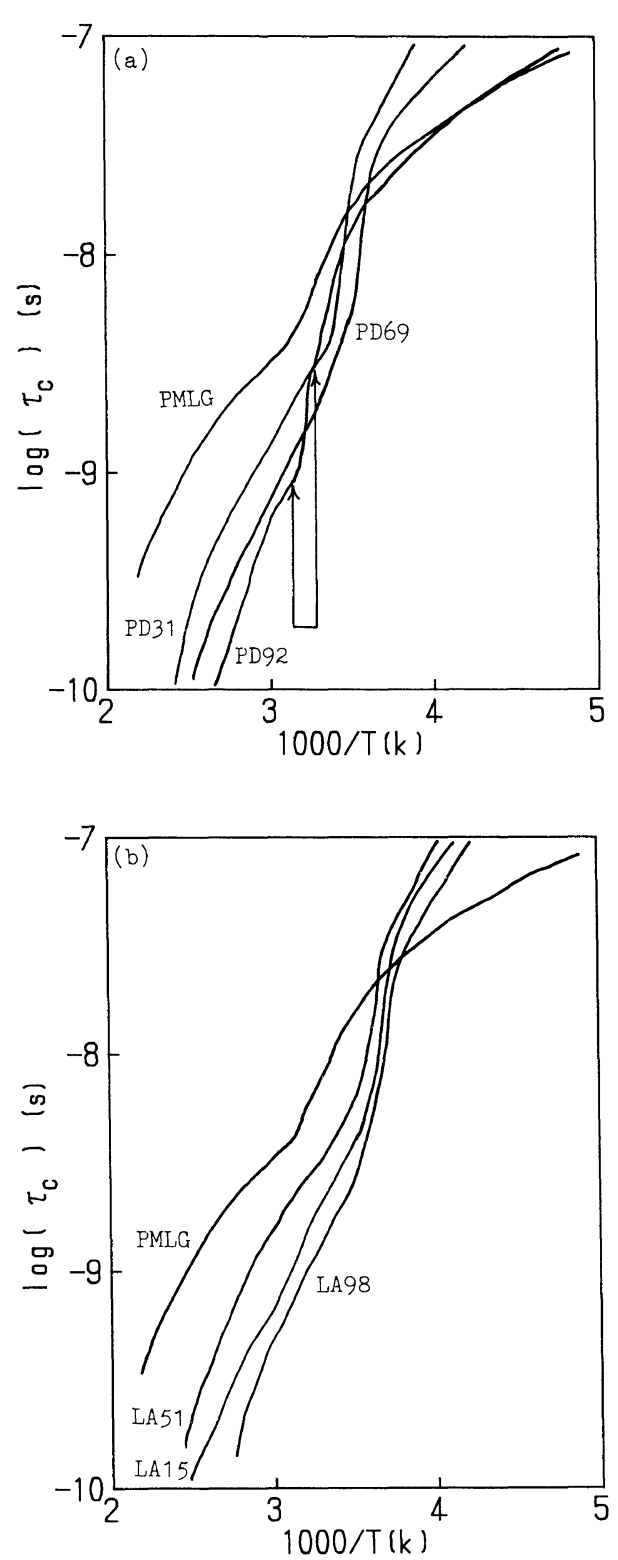

$\tau_{\mathrm{c}}$ occurs and will be disscussed below. As seen below and above a glass transition in amorphous polymers, ${ }^{9}$ motional modes in lower and higher temperature processes were attributed to the small amplitude oscillation of spin-probe and jumps between cavities (free volume), respectively. These conclusions were made on the basis of the well known fact that

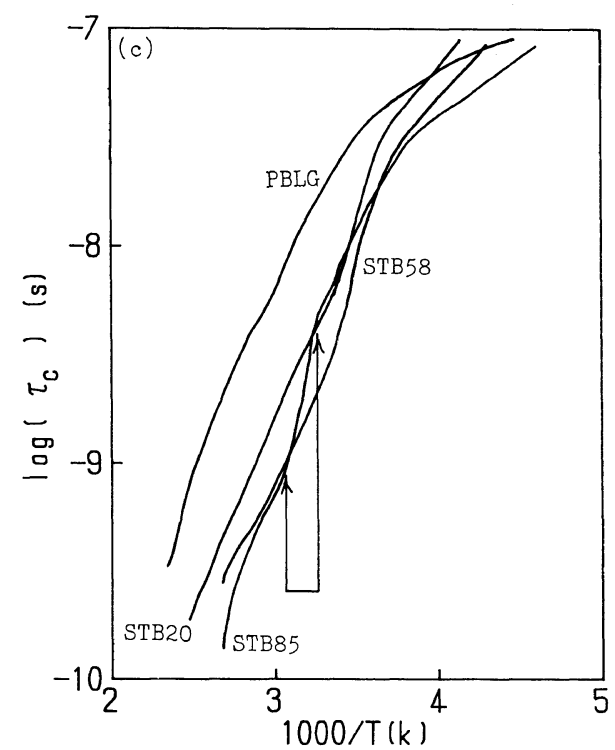

Figure 4. Temperature dependence of $\tau_{\mathrm{c}}$ for PD (a), LA (b), and STB (c) copolymers.

motional behavior of side chains of poly(glutamate)s showing the glass-like transition which is similar to that of amorphous polymers. ${ }^{8,13,14}$ In each copolymer series, there is a tendency that $\tau_{\mathrm{c}}$ decreases as D.S. increases in higher temperature region, indicating the increase of side chain mobility. Around $1000 / T(\mathrm{~K})$ of 3.0 to 3.5 (333.3 to $285.7 \mathrm{~K})$, PD92 and STB85 have longer $\tau_{c}$ than that for copolymers with smaller D.S., whereas LA98 has the shortest $\tau_{\mathrm{c}}$ in LA copolymer series. Discussion on this noticeable result is given below.

To investigate the anisotropy of rotational motion of TEMPOL in the side chain region, anisotropic correlation times $\tau_{\|}$and $\tau_{\perp}$ of PD and LA copolymers, which were estimated by the method described above, are plotted in Figure 5. It is clearly seen that in all samples, TEMPOL undergoes anisotropic motion in spite of spherical molecular shape. ${ }^{15}$ PMLG has about 10 times larger $\tau_{\perp}$ than $\tau_{\|}$even at the highest temperature. However, in the copolymers, difference between $\tau_{\|}$and $\tau_{\perp}$ is much smaller, and almost vanishes in the higher temperature region for $\mathrm{PD}$ and LA 

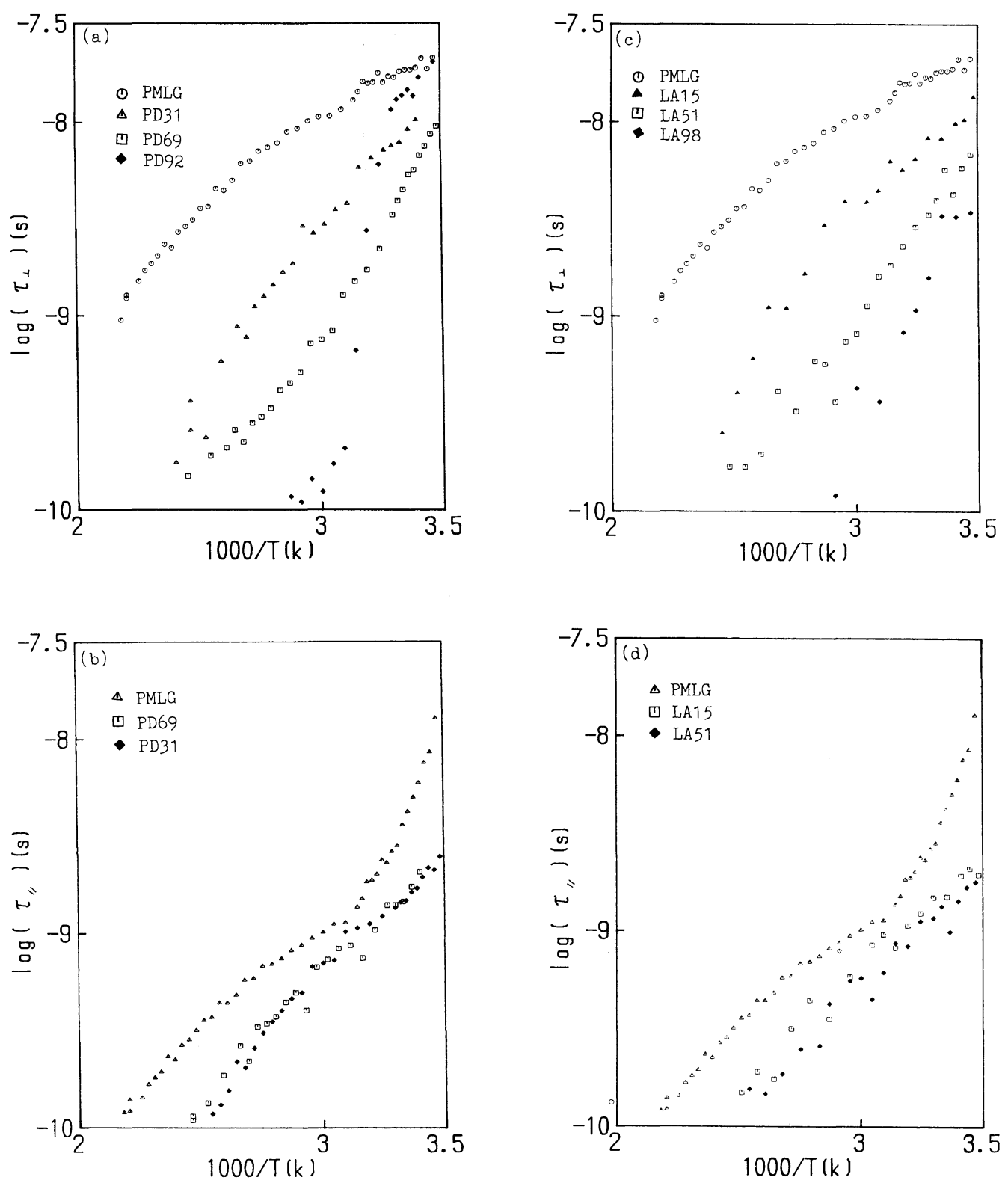

series. In these copolymers, $\tau_{\perp}$ decreases in the higher temperature region as the D.S. increases, although $\tau_{\|}$shows no dependence on D.S. It is suggested that $\tau_{\perp}$ is influenced more strongly by the motional state of host polymer, since the rotation about minor axis of symmetric rotor needs more space than that about the major axis.

Figure 5. Temperature dependence of anisotropic rotational correlation times. $\tau_{\perp}$ (a) and $\tau_{\|}$(b) for PD, $\tau_{\perp}$ (c) and $\tau_{\|}(\mathrm{d})$ for LA copolymers, respectively.

The ratios of two correlation times $N=\tau_{\perp} / \tau_{\|}$ are plotted against the reciprocal of temperature in Figure 6. $N$ is clearly different between copolymers and PMLG. Copolymers have $N$ of $c a .1$ to 5, whereas PMLG has $c a$. 10. In 

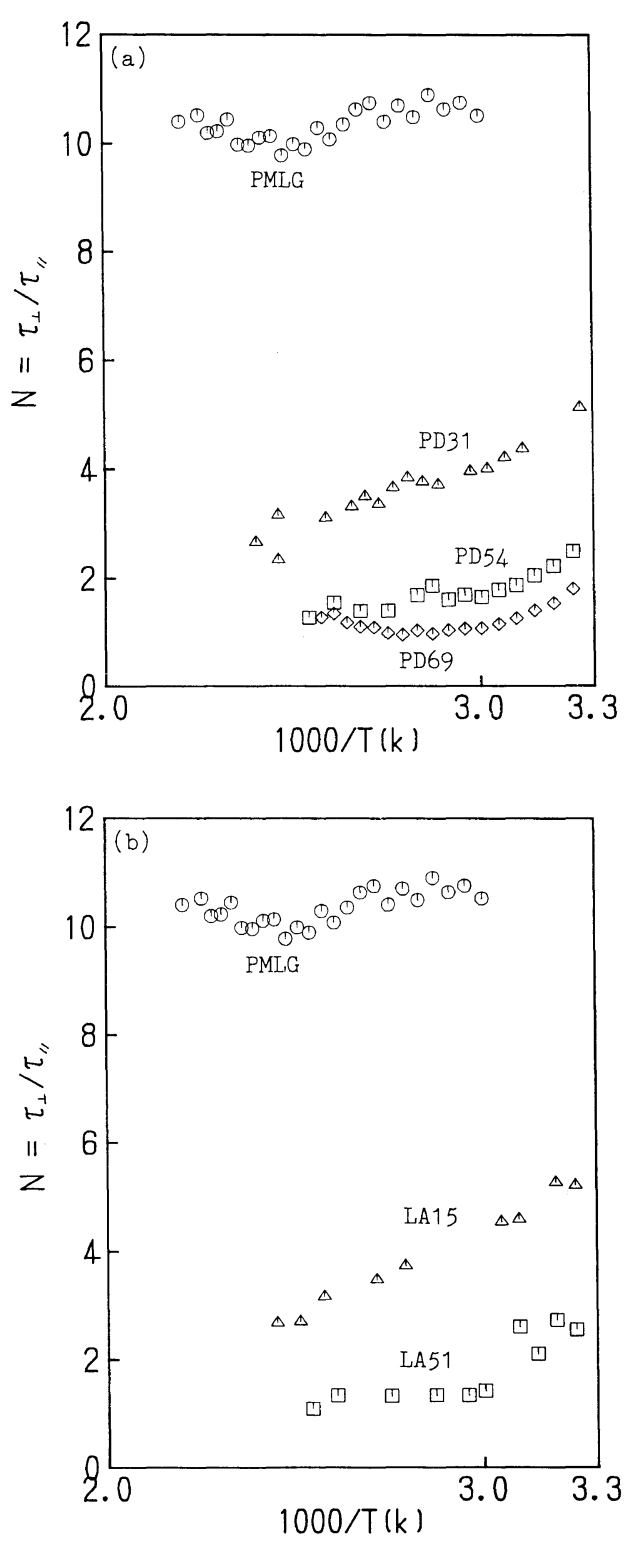

Figure 6. Temperature dependence of $N=\tau_{\perp} / \tau_{\|}$for PD (a) and LA (b) copolymers, respectively.

copolymers, $N$ becomes smaller with increasing D.S. If the spin-probe has completely spherical shape and the environment is isotropic, $N$ should be unity. The reason why $N$ depends on temperature is not clear. However, one possibility is proposed as follows. The relation to estimate the rotational correlation times is derived for an ellipsoid with a smooth surface in isotropic and continuous media. In an actual case, spin-probe has some 'protuberance' and does not have ideally ellipsoidal shape. In the present case, the environment of spin-probes consists of side chains sticking out from $\alpha$-helical main chains, which may have preferable direction. These situations are much different from those of an ideal case. ${ }^{16}$ It is considered that the actual rotational ellipsoid consists of spin-probe and the 'solvent' tagged around the former. If there is some additional interaction between a spin-probe and host polymer, such as hydrogen bonding, ${ }^{17}$ the shape of the 'effective' ellipsoid may be changed. As a result, the anisotropy of spin-probe in each polymer is different and changes depending on temperature.

Small $N$ for copolymers indicates that the side chain of copolymers has much freedom of taking many conformations and undergoes fast motion as to average out the interactions between TEMPOL and the side chain. As a result, the side chain region seems to be an isotropic liquid like media. On the other hand, large $N$ for PMLG indicates that the motional state of side chain region is far from the isotropic liquid.

There is a tendency that $N$ becomes smaller for both PD and LA copolymer series as D.S. increases in the higher temperature region, the values of $N$ being around unity for copolymers with D.S. of more than $50 \%$. These facts indicate that the motional state of long alkyl groups at the high D.S. is similar to that of isotropic liquid in the copolymers of PD and LA series.

\section{Dependence of Spin-Probe Motion on Side Chain Length}

As pointed out in the previous section, PD92 $(n=15)$ and STB85 $(n=18)$, which have the highest D.S. in each copolymer series, have higher $T_{50 G}$ than that for samples with intermediate D.S. (see Figure 3) as reported also for ST83, ${ }^{5}$ whereas LA98 $(n=12)$ does not 


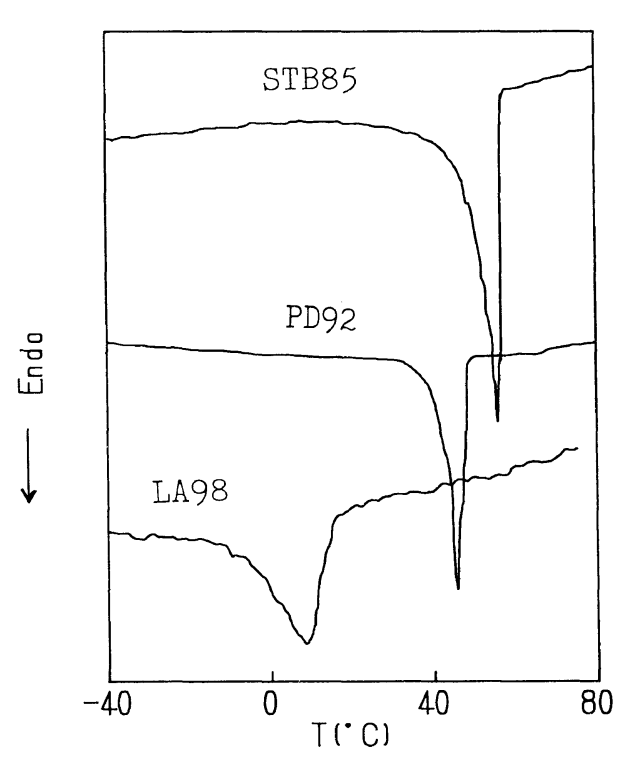

Figure 7. DSC thermograms of PD92, LA98, and STB85 in the heating process.

have such a higher $T_{50 \mathrm{G}}$. Furthermore, $\tau_{\mathrm{c}}$ for PD92 and STB85 decreases suddenly at around the temperature indicated by two arrows (see Figure 4) and becomes smallest in the respective copolymer series above this temperature. Similar behavior was reported for ST83 $(n=18) .{ }^{5}$ However, LA98 does not show such a decrease (see also Figures 5(a) and (b)). The previous work for the ST copolymer ${ }^{5}$ indicated that spin-probe motion is constrained by crystallites consisted of long alkyl groups at high D.S. Therefore, results for the present copolymers imply that PD92 $(n=15)$ and STB85 $(n=18)$ have high crystallinity that restricts spin-probe motion, whereas LA98 $(n=12)$ does not have such high crystallinity.

The latter conclusion was supported by DSC measurements. Figure 7 shows DSC thermograms for PD92, LA98 and STB85. An endothermic peak, which corresponds to the melting of side chain crystalline, is observed. ${ }^{1}$ In the other samples which are not shown here, the peak temperature decreases and peak profile becomes broader as the D.S. decreases. PD92 and STB85 have relatively sharp endothermic peaks, whereas LA98 has a much broader one. This implies that the size and/or orderliness of crystallite are much lower in LA98.

\section{Effects of Different Short Side Chain Units on Motional State of Long Alkyl Chains}

It is of interest to compare $\tau_{\perp}$ of ST and STB series at comparable D.S. to see the influence of different short side chains in MLG and BLG on the long alkyl chain motion. First of all, $\tau_{\perp}$ for the homopolymers with short side chains, PMLG and PBLG, is compared in Figure 8(a). These polymers show different temperature dependences of $\tau_{\perp}$; PMLG has a much smaller slope than PBLG. These tendencies are also seen in the second moment-temperature curve for ${ }^{1} \mathrm{H}$ broad line NMR spectra in the temperature region where large scale motion of side chain occurs. ${ }^{8,18,19}$ This similarity is evidence that spin-probe motion above room temperature reflects such motional process. Figures 8(b) - (d) show the results for copolymers. At higher D.S. (Figures 8(c), (d)), $\tau_{\perp}$ values for two copolymers are almost similar in spite of having short side chains with very different structures. At lower D.S. (Figure 8(b)), ST25 and STB20 still keep a tendency similar to PMLG and PBLG, respectively. The effects of short side chains on spin-probe motion is seen only in copolymers with small D.S. These results imply that the motional state of long stearyl group $(n=18)$ is not influenced by the short side chains at higher D.S.

\section{CONCLUDING REMARKS}

In all series of LA, PD, and STB copolymers, there is a tendency that side chain motion becomes rapid with increasing degree of substitution of long alkyl side chain (D.S.). For the samples with highest D.S., PD92 and STB85, a sudden decrease in the rotational correlation time was observed around the melting point of side chain crystalline. For LA copolymers, such a decrease is not observed 

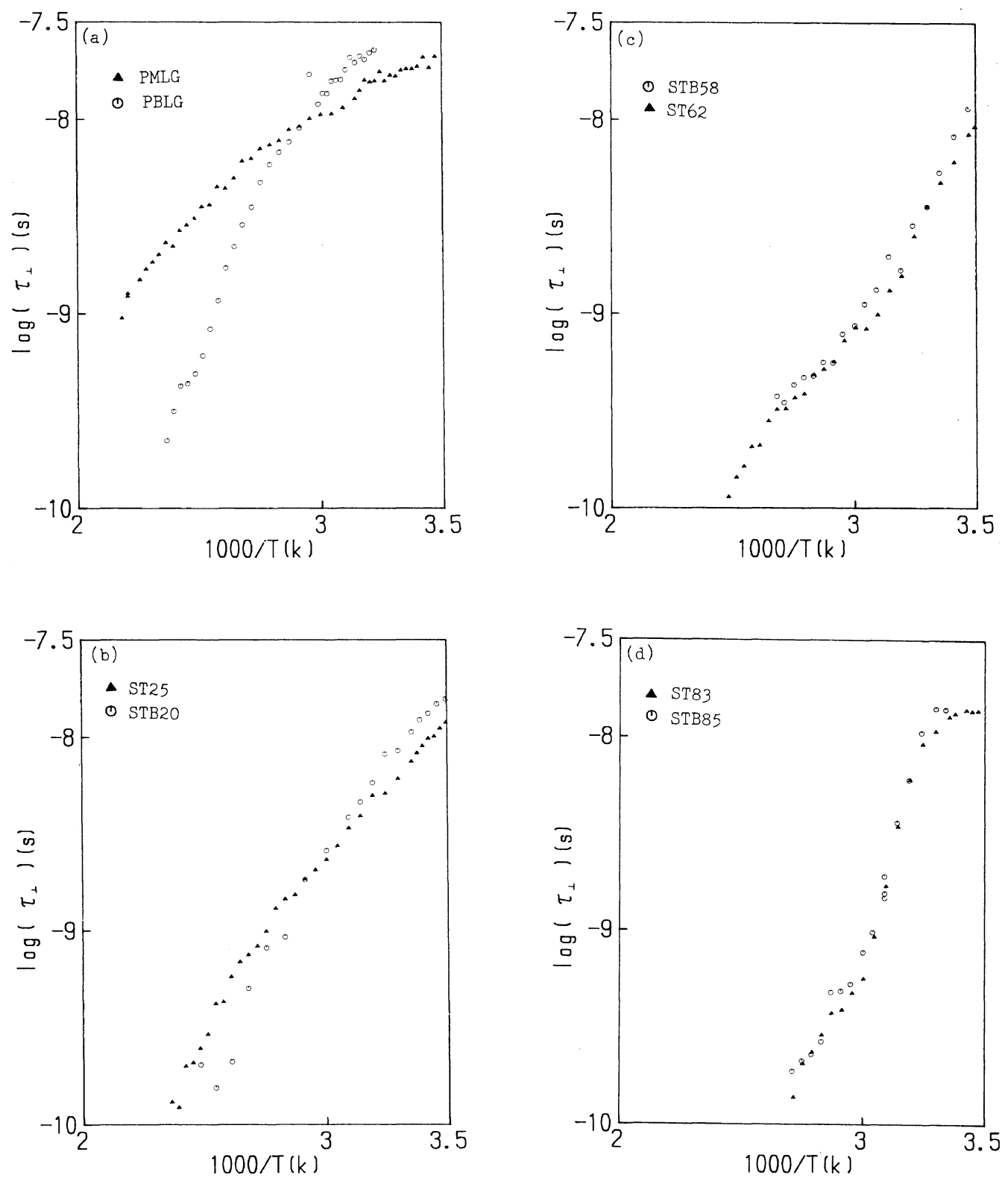

even at the highest D.S., indicating that the side chain crystalline is not large enough to restrict the probe motion.

On the effect of short side chain on the motion of long alkyl side chain, the motional state for STB and ST copolymers with comparable D.S. was quite similar to each other except for small D.S. region. This means

Figure 8. Temperature dependence of $\tau_{\perp}$ for PMLG and PBLG (a), for ST20 and STB25 (b), for ST62 and STB58 (c), and for ST83 and STB85 (d).

that motion of long alkyl side chain with $n=18$ is not affected by short side chains at higher D.S. These results agree with the previous results for ST copolymers that long alkyl groups undegoes motion with no constraint 
from the inner part of side chain region composed of short side chains and inner portion of STLG side chains, and behave as paraffin like molecules.

Acknowledgments. The authors wish to express their gratitude to $\mathrm{Mr}$. F. Ishii of Hokkaido University for his valuable advice. This work was supported by the Ministry of Education, Science, and Culture of Japan.

\section{REFERENCES}

1. J. Watanabe, H. Ono, I. Uematsu, and A. Abe, Macromolecules, 18, 2141 (1985).

2. J. Watanabe, M. Goto, and T. Nagase, Macromolecules, 20, 298 (1987).

3. J. Watanabe and T. Nagase, Polym. J., 19, 781 (1987).

4. T. Yamanobe, M. Tsukahara, T. Komoto, J. Watanabe, I. Ando, I. Uematsu, K. Deguchi, T. Fujito, and M. Imanari, Macromolecules, 21, 48 (1988).

5. M. Yamaguchi and A. Tsutsumi, Polym. J., 22, 781 (1990).

6. L. J. Berliner (Ed.), "Spin Labeling," Theory and Applications, Academic Press, New York, N.Y., 1976.
7. P. Meurisse, C. Friedrich, M. Devolaitzky, F. Laupretre, C. Noel, and L. Monnerie, Macromolecules, 17, 72 (1984).

8. A. Tsutsumi, K. Hikichi, T. Takahashi, Y. Yamashita, N. Matsushima, M. Kanake, and M. Kaneko, J. Macromol. Sci. Phys., B8, 413 (1973).

9. N. Kusumoto, in "Molecular Motion in Polymer by ESR,” R. F. Boyer and S. E. Keinath, Ed., Harwood, Chur, 1980.

10. J. H. Freed, J. Chem. Phys., 41, 2077 (1964).

11. A. L. Kovarskii, A. M. Vasserman, and A. L. Buchachenko, Vyskomol. Soyedin., Ser. A, 13, 1647 (1971).

12. S. A. Goldman, G. V. Bruno, and J. H. Freed, $J$. Chem. Phys., 76, 1858 (1972).

13. A. Tsutsumi, Jpn. J. Appl. Phys., 9, 1125 (1970).

14. Y. Yamashita, A. Tsutsumi, K. Hikichi, and M. Kaneko, Polym. J., 8, 114 (1976).

15. A. M. Vasserman, A. N. Kuznetsov, A. L. Kovarskii, and A. L. Bulachenko, J. Struct. Chem., 12, 555 (1971).

16. D. Kivelson, in "Rotational Dynamics of Small and Macromlecules," Th. Dorfmuler, R. Pecora, Ed., Springer-Verlag, Berlin, 1986, p 1.

17. Z. Veksli and W. G. Miller, Macromolecules, 10, 1245 (1977).

18. K. Hikichi, K. Saito, M. Kaneko, and J. Furuichi, J. Phys. Soc. Jpn., 19, 557 (1964).

19. Y. Yamashita, A. Tsutsumi, K. Hikichi, and M. Kaneko, Polym. J., 8, 114 (1975). 